\title{
New Ways of Deliberating Online: An Empirical Comparison of Network and Threaded Interfaces for Online Discussion
}

\author{
Anna De Liddo and Simon Buckingham Shum \\ Knowledge Media Institute, The Open University, Walton Hall \\ MK76AA, Milton Keynes, United Kingdom \\ \{anna.deliddo, simon. buckinghum. shum\} @open.ac.uk
}

\begin{abstract}
One of the Web's most phenomenal impacts has been its capacity to connect and harness the ideas of many people seeking to tackle a problem. Social media appear to have played specific and significant roles in helping communities form and mobilize, even to the level of political uprisings. Nevertheless the online dialogue spaces we see on the Web today are often repurposed social networks that offer no insight into the logical structure of the ideas, such as the coherence or evidential basis of an argument. This hampers both quality of citizen participation and effective assessment of the public debate. We report on an exploratory study in which we observed users interaction with a new tool for online deliberation and compared network and threaded visualizations of arguments. Results of the study suggest that network visualization of arguments can effectively improve online debate by facilitating higher-level inferences and making the debate more engaging and fun.
\end{abstract}

Keywords: Argumentation, Computer Supported Argument Visualisation (CSAV), Online Deliberation, Collective Intelligence.

\section{Introduction}

Online debate through social media is increasingly used to promote citizens' engagement in policy and decision-making. While common social media websites reach increasing number of users all around the globe, and successfully respond to the demand of scaling up citizen access to public debate [1], the way in which those tools support discourse structuring, representation and analysis remains quite limited [2,3]. This hampers the very quality of citizen participation to the public discourse and undermines the impact of citizen contributions to e-government and e-participation processes. We argue that one of the main barriers to quality e-participation to public debate is the lack of support for discourse reading and understanding. It is hardly possible for citizens to quickly grasp the main issues involved in an online debate, understand the articulation of the arguments presented, and discovering emerging synergies and conflicts. 
In this paper we present results of an exploratory study, which suggests that network visualization of arguments can effectively improve the way in which users read and understand online discourse in at least two ways:

- by facilitating higher-level inferences of how the online discourse contents relate; and

- by supporting the identification of argumentation chains, synergies and contrasts within the discourse.

Moreover, we observed that the dynamic animation of networks adds an element of fun and excitement to arguments exploration. This may play an important role in promoting users' engagements with the online debate, especially crucial for motivating younger citizens.

In the following we detail the results of the study, starting from describing background knowledge and motivating the research in the CSAV (Computer Supported Argument Visualization) and Online Deliberation literature (section 2). We describe the research question and method (section 3), report on the user study (design, and experimentation setting), and describe the tool used to investigate the research question (section 4). Finally we discuss the data analysis and interpretation and present main research findings (Section 5). We conclude by outlining potential applications and future research (section 6).

\section{Background and Motivation}

\subsection{Background Knowledge: Computer Supported Argument Visualization for Online Deliberation}

The rationale and motivation for this work have roots at the intersection of Computer Supported Argument Visualization (CSAV) and Online Deliberation (OD) research, which studies how computer mediated communication can be combined with argumentation theory to enhance public deliberation. We aim to investigate how multidimensional communication flows (between people, environments, time, topics and points of views) can be translated into coherent discourse and eventually lead groups to better understanding "wicked problems" [4]. Wicked problems are undetermined and complex by nature, and they require deliberative discussions and argumentation to be better understood and tackled. Supporting better understanding and deliberating is the first steps toward the development of informed participation to policy and decision-making, and it therefore sits at the art of e-participation research.

Computer Supported Cooperative Work (CSCW) and Computer supported Collaborative Argumentation have been recognized as two major areas of research that provide support for e-consultation (pp 53, [5]). Many projects prove the extent of interest and research in this area and its relevance to develop new technologies for eparticipation (for a review of argumentation tools for e-participations see [6, 7]). CSAV tools have proved to encourage debate and deliberation by citizens on public issues and have been applied f.i for policy formulation to provide a visual medium by which citizens can follow and join in public debates on policy issues [8]. 
Tambouris et al. even identify CSAV between the key enabling characteristics to consider when assessing eParticipation projects and tools [9].

Computer Supported Argument Visualization (CSAV) aims to augment the personal and collective ability of users to explore complex problems, make sense of difference in viewpoints, discuss options, and reflect on the implication of those by analyzing and constructing arguments.

CSAV is a research field which has its roots that span several disciplines, such as Philosophy, HCI, hypertext, CMC, and several domain applications, such as design, education, law and public policy [10]. The idea that people can augment their human intellect and their capability to "comprehend and find solution to complex-problem solving situations" by manipulating explicit and externalized "concept structures" dates to Douglas Engelbart [11]. Concept Mapping [12] and Argument Mapping [13] have been applied to support education and critical thinking and several authors identified "visualization" as an important but unexploited dimension for refining and communicating one's thoughts [14]. Argumentation and the associated technological support for argument analysis and construction, have been widely investigated in the two decades from the 70ies and 90ies, when first class researchers in the hypertext community developed prototype tools (between others NoteCards [15], gIBIS [13] and AAA [16]) and carried out pilot studies to explore advantages and shortcoming of this approach [17]

In design rationale studies several limitations have been identified which highlights the difficulty to use computer supported argumentation for design practice, particularly related to the conceptual and time overheads in fragmenting and structuring thoughts before communication. Fisher and al. [18] suggest that there are many limitations that need to be overcome to make argumentation (and argumentation tools) serve design; and these concerns have been echoed in [19].

Nonetheless, in another domains such as Technology Enhanced Learning, Public engagement and e-Participation the interest in argumentation and CSAV has persistently been followed, also encouraged by the recognized advantages of this approach in term of support to reflection, knowledge construction and learning [20]. Example tools for large-scale deliberation are currently classified as e-participation, edemocracy or e-government systems [21, 22] some of which uses CSAV as a way to structure and facilitate citizens consultation [8] and large scale participation to public debates [23].

An established research literature documents the advantages of making the structure and status of a dialogue or debate more visible [10]. Scheuer and al. [24] review the state of the art of computer-supported argumentation, which proves the extensive interest and production of research and technology in this field. Scheuer and al. present a rich overview of both the types of argument representations, as well as the variety of interaction designs and ontologies to support argumentation. Several empirical studies are also presented that have been carried out to assess various argumentation systems in different domains. While many of these systems are aimed to support argumentation in a learning context [25](e.g Belvedere [26] and ARGUNAUTUT [27]); many others have been designed to support argumentation in 
other fields such as law (Carneades [28] and Rationale [29]; science (SenseMaker [30], WISE [31]) and decision making (QuestMap, Compendium [32, 33]).

We are interested in this last category of argumentation systems and their Web successors which have been designed to support e-democracy and e-participation by promoting citizen engagements in decision-making throughout online discourse processes. These are specifically designed collective intelligence infrastructures and large-scale argumentation systems to structure informal online discussion as argumentation processes and use CSAV to makes visually explicit users' lines of reasoning and (dis)agreements [34]. Naturally, the use of semantic networks to provide computational intelligence has now converged with Web Science, resulting in Web 2.0 Argumentation [35] and a semantic web standards-based Argument Web [36].

\subsection{Research Gap and Motivation}

Many of these CSAV tools are now available, exhibiting a wide mix of network visualizations and more conventional threaded renderings. Whereas many systems use a common ontology to represent discourse element (such as IBIS [4] or its more design oriented version QOC [37], when it gets to argument visualization there seems to be no agreement on what is the best interface for argument visualization. Deliberatorium uses a linear-threaded visualization [3], while Cohere [35] supports a graph visualization of the online discourse. Debategraph ${ }^{1}$ and CoPe_it! [38] enable linear, threaded and graph views. However, to date, we have not yet found a systematic evaluation of the merits or otherwise of CSAV vs. threaded interfaces.

In addition to this, the suitability of graphs as medium to support argumentation has been questioned by some, for three main reasons: complexity of the ontology, number of participants and domain of application. Some researchers argue that the bigger the number of participants to the discussion, and the higher the complexity of the discourse ontology, more clumsy and less usable graph visualizations become (pp.53 [24]). In addition, there seems to be resistance to the use of graph visualization of arguments in certain domains of application. Hair [39] reports on a study in the legal field in which lawyers have expressed a strong preference in favour of linearthreaded text representations of arguments compared to network visualization.

It therefore remains unclear what are the advantages and affordances of different graphical representation of arguments to support online discussion and large-scale deliberation. This motivates the research presented in this paper.

An exploratory user study was carried out to compare network visualizations and threaded discussions as the two most commonly used interfaces for argument visualization. We aimed to assess which one of these two visualizations better support online discourse in two main tasks: argumentation reading and understanding. Argumentation reading and understanding are at the cornerstone of effective participation to online discourse, and they also consist of the most prominent users interaction modality. In fact, the internet usage pattern of "participation inequality"

\footnotetext{
${ }^{1}$ DebateGraph 2013: Web tool for the visualization of idea and debates http: / / debategraph.org
} 
[40] confirms that typically, only a very small percentage of users contributes to a website, compared to vast majority who consume. So, while browsing, searching, reading and writing clearly cannot be divorced from each other, the focus of the study reported here is not on the authoring process, but on the experience and performance of the majority of users who will be reading and searching the online deliberation platforms.

\section{$3 \quad$ Research Question and Methodology}

Does an interactive, self-organizing network visualization of arguments provide advantages over a more conventional threaded interface for reading and search? The following research questions was formulated:

$R Q:$ What are the recognized advantages of threaded and network visualization of arguments for supporting online discourse reading and understanding?

A grounded theory analysis has been conducted to study the use of different graphical interfaces for the representation of arguments, and to assess to what extend these affect the way in which users read and understand the online discourse. Grounded Theory is a qualitative research method which has been widely used in HCI to provide insights on people's views, behaviors, understanding and experience with technology [41]. Very recently the discussion has been revamped on what are the nuances and innovation to the method that may help to respond to the increasing request and diffusion of qualitative research studies in HCI [42].

We took a 'constructivist' stance to grounded theory which aims at creating a description of the context rather than discovering a description of it (objectivist approach). We started by keeping a Glaserian approach to data analysis and applied open coding of the data trying not to be influenced by prior ideas or extant theories. The initial open coding phase was performed on transcripts of the screencast video of users' interaction in the experimentation. Video clips and transcripts were analyzed and coded in an unfocused manner. While reiterating the coding from one user experimentation to the following, we moved progressively to a more focused coding in which specific concepts and themes started emerging. From this analysis several categories emerged which provided the building blocks of our findings, and the main pillars of our narrative of the analyzed phenomenon.

The coding process generated over 350 codes, which have been edited and merged in several revising cycles, and then have been grouped into main sub-categories ( 23 in total) and higher-level categories of analysis ( 9 in total). The main higher-level categories emerged provided a taxonomy of the main components/variables which effect users capabilities to read and comprehend online argumentative discussion. The 9 categories have then been ordered on the base of what were more significant in terms of three metrics: number of quotations per code (N. of Videoclips/Quotation), number of participants who experienced the event captures by the code $(\mathrm{N}$. of People affected), and time of interaction per code. As a result three main higher-level code categories emerged as key factors affecting users interaction and these have been used as metrics to access users performance with the two interfaces (see section 5). 
We then developed a narrative around these 3 emerging themes, but also around events and findings, rooted in the in depth observation and analysis of the interaction. This allowed us to draw some conclusions on what are the recognized advantages of threaded and network visualization of arguments to support online discourse reading and understanding.

\section{User Study}

\subsection{Goal, Design and Participants}

We studied an heterogeneous group of researchers and practitioners in Higher Education engaged in the use of a collective intelligence tool for collaborative argumentative discourse and knowledge construction. An exploratory user study was run to observe users' performance under three information-seeking tasks, and compare their performances using two different user interfaces for arguments visualization (threaded vs network visualization of arguments). Participants were divided in two groups of 5 (Group1 and Group2). The 10 subjects were drawn from the members of different Open University departments, so with widely mixed IT expertise. They were randomly allocated to the two different groups, but we verified post-hoc that IT expert/non ex-pert ratio in each group was approximately the same. The median age was 40 (with range from 32 to 48) with the majority of users being either native or near-native English speakers.

\subsection{Online Environment: The Evidence Hub}

The online discussion tool used throughout the study was the Evidence Hub system[43]. The Evidence Hub is a collective intelligence and online deliberation tool to support argumentative knowledge construction by crowdsourcing contributions of issues, potential solutions, research claims and the related evidence in favor or against those. The Evidence Hub is particularly oriented to support community of practitioners to build evidence-based knowledge about specific key challenges that are set up to the community. This key challenges can then be addressed by tackling specific sub issues, that the community contribute to add to the system. Each sub issue can then be tackled by proposing solutions to it, or by sharing specific research claims that can help tackling the issue. Finally potential solutions and research claims can be debated by the community by advancing evidence in favor or against those and by providing relative resources backing them up. By scaffolding users contributions in this way, the Evidence Hub aim to effectively crowdsource and support large-scale deliberation in e-democracy and decision-making processes. To allow comparison between users interaction with two graphical representations of arguments, two different versions of the Evidence Hub have been set up which used different user interfaces for arguments visualization. The two versions of the Evidence Hub pointed at the same database to ensure that participants in the two groups would receive the same quantity and type of information. 


\subsection{The Linear-Threaded Interface for Arguments Visualization}

The linear-threaded interface was used by Group1 (Figure 1). This interface for arguments visualization is similar to the most common threaded online discussion interfaces to support argumentative discourse activities. It consists of a classical threaded visualization in which issues set the focus of discussion (title of the page in Figure 1), and then potential solutions are listed below (light bulb icons are placed before each solution). Each solution can then be expanded on demand. The progressive indentation of text shows the supporting and challenging evidence for a solution, and the re-sources supporting evidence (Figure 1).

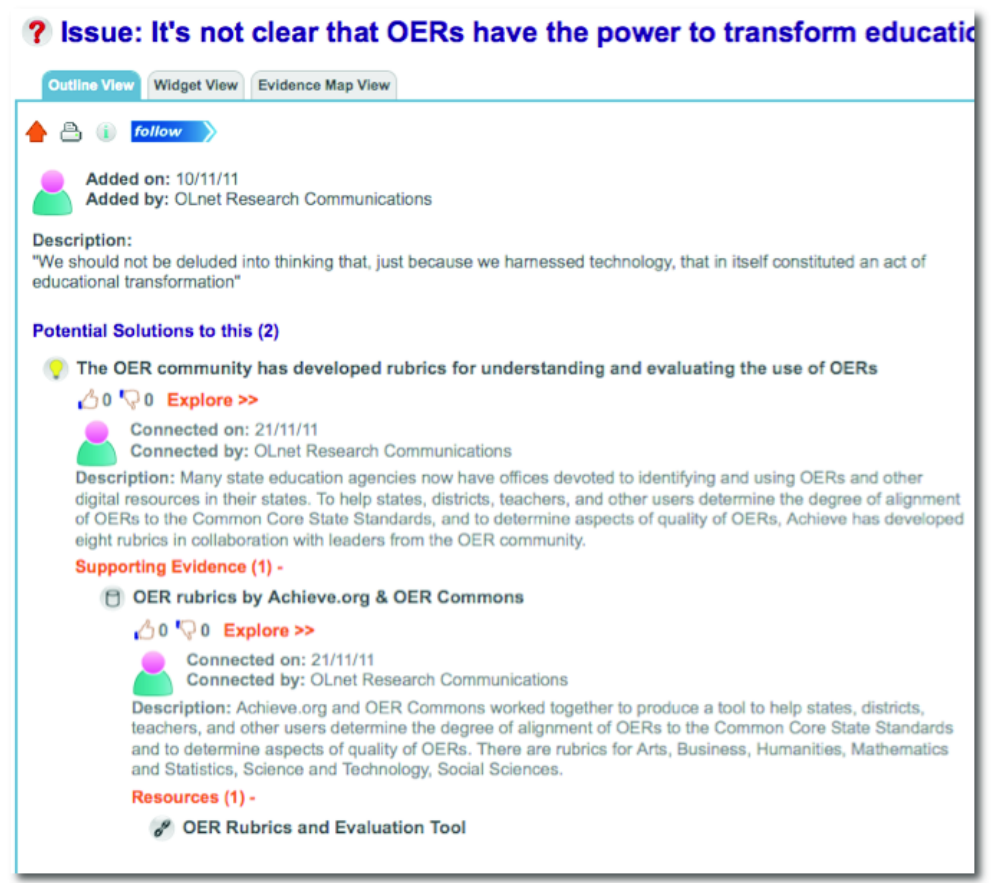

Fig. 1. Linear-threaded Interface for Arguments Visualization

\subsection{The Network Interface for Arguments Visualization}

The network interface was used by Group 2: This consists of an argument map built by following the modified IBIS, PHI model of arguments [44]. Each statement added to the discussion is here represented as a node in a semantic graph structure (Figure 2). In this visualization, node icons and colors represent the rhetorical role of the statement in the discussion: issues are characterized by a red question mark icon in a dark turquoise node (yellow in Figure 2 because the node is selected); solutions are distinguished by a light bulb icon (see light green nodes), while pro and con are characterized by purple nodes and are connected with a green link or red link to respectively indicate "supporting" or "challenging" relations. 
The network visualization of the Evidence Hub is build with a Java Applet, which lays out the nodes dynamically, and following a gravitational algorithm. This adds a specific animation component to most common argument network visualization tools.

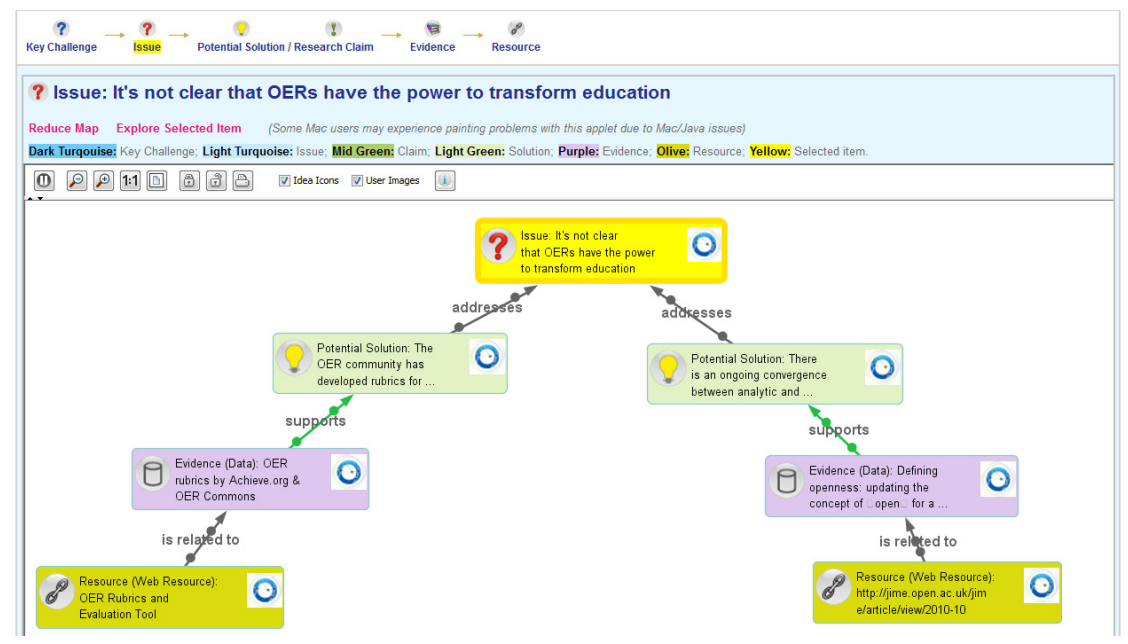

Fig. 2. Screenshot of the Network Interface for Arguments Visualization

\section{$5 \quad$ Data Analysis and Results}

The experimentation design aimed to provide an answer to the RQ by focusing on three specific information-seeking tasks (for issues of space we skip detailed description of the tasks):

1. Identifying solutions to an issue (Task1)

2. Identifying synergies between solutions (Task 2)

3. Identifying contrasts in the wider debate (Task 3)

The tasks lasted about 2 to 3 minute and required users to scan 1-3 Webpages (about 1500 - 4000 words) depending on the task.

The three higher-level class of codes emerged from the grounded theory analysis of the user-interaction videos were used as metrics to compare the user's performance across the three tasks. The metrics are: Task Accomplishment; Data Model Interpretation and Emotional Reactions to the two interfaces. From the grounded theory analysis, these metrics have been found to represent the most relevant factors affecting interaction and therefore have been used to assess users' performance with the two interfaces. In the following we analyze and compare users' performance by focusing on each of these metrics and providing users code to motivate our understanding. 


\subsection{Task Accomplishment}

Four main sub-categories of accomplishment emerged form the coding: Accomplished easily, Accomplish but incorrect, Not accomplished, and Give Up the task. From the analysis of the quotations and of the screen capture video of the interaction we found that the main reason of failure was task's understanding. Most interaction events coded as "Accomplished but incorrect" were reported in Group 1. The linear structure of the interface is not designed to help interconnecting and comparing content and this has proved to be a burden to the user. As a consequence of this limitation, users who interacted with the linear interface focused more on issues of content's style and validity, rather than on the argumentation process, which lead to digressions and incorrect responses. On the contrary the interaction events coded as "Accomplished easily" were mainly experienced in Group 2. This is mainly due to the visual hints provided by the network representation. Specifically, links labels and colours seemed particularly predominant in determining success. We noticed that, the users who accomplished the task easily usually relayed on links colours and label, and paid less attention to the iconography of the nodes. The number of links (connections density) also proved to be a very effective way to provide answers to the task.

\subsection{Comparing Data Model Interpretation}

In order to compare linear and network visualization of arguments in term of how they supported Data Model Interpretation (DMI) we first identified the emerging codes related to DMI and merged them under the following 5 main classes (1. get the model right, 2. get the categories right, 3. get the model but is unsure, 4. misinterpret categories, 5 does not get the model at all). We then analysed the interaction events and quotations for each main class.

Results show that DMI was better supported by network visualization of arguments. Category misinterpretation and uncertainty in data model interpretation tend to occur more frequently in Group1 than in Group2, while network visualization of arguments seems to support a complete and correct understanding of both categories and data model. Findings suggest that one possible motivation for this is that argument maps provides examples of how the content of discussion is interpreted in the data model supported by the Evidence hub. In this way users can learn by example ("let's see what other people have put under this category" - P8) and at each exploration of a new argument map they reinforce their understanding of the data model ("this is my solution and this evidence support the solution, and these two nodes challenges it...the clue is the red "challenges" link-P7)".

\subsection{Comparing Emotional Reactions}

Emotional reaction to the graphical representation of arguments is another emerg-ing category that we used to measure users satisfaction and emotional attitude toward the two proposed interfaces. Results show that a general sense of surprice and positiv-ity toward the network visualization was recorded, which easily sparks into linkeness and 
even exhitment (“ahhh so fabulous!"-P7; "wow this is amazing!"-P6). This postive emotional reaction also seems to be associated to an increase of user' s confidence with the tool ("I feel confident, I'm pretty sure this is the answer"-P7). The main object of positivity toward the network visualizations was the self-arranging graph applet. The Applet presents argument maps as floating nodes and edges, slowly moving and arranging on the screen ("it is like a jelly, it is so fantastic!"-P7; "it is all shifting! It is interesting... I quite like that!"-P8). Movements provoked surprise and excitement and it was also recognized as a useful information feedback. By looking at the floating network, users understood that the map was not static and they could move nodes around and play with it. Users reported that they mostly enjoy this feature. In summary, findings suggest that: moving arranging, zooming and pinning are the network features which most augment users confidence and satisfaction with the tool. On the contrary emotional reactions to the linear interface for arguments visualization were general skepticism and dissatisfaction, and sometime also decayed to confusion and feeling lost ("I think I am lost"-P1; "I am now really annoyed.. because I haven't worked out how to do it" "that is the all page I am looking at?...ohhh ok I give up!'”-P5). Some users explicitly identified the cause of their frustration and said that they needed "too many clicks" to seek information and frequent "change of context" which often provoked disorientation.

\section{Contributions and Future Research}

The grounded theory analysis of the experimentation's video showed that different graphical interfaces for the representation of arguments strongly affect the way in which users read and understand the online discourse. Network-like representations and visual hints such as network structure, iconography, links' labels and colors seem to facilitate the identification of argumentation chains, thus supporting indirect connection and higher-level inferences of how the content connects. The results show that data model interpretation is also improved by argument visualization. Notably, exposing the data model in form of argument maps appears to enable learning-byexample mechanisms, whereby users reinforce their understanding of the data as they navigate through the user interface. Finally there is an element of fun and excitement associated to dynamic network visualization of arguments. This may suggest that this type of arguments visualization could be useful for e-participation processes in which the element of "play" and positive emotional reaction are key factors to success. This paper shows promising results on the capability of network visualization of arguments to support reading and sensemaking of online discourse activity. Of course the challenge for e-participation is: how can we move from lab experiments to real world setting and scale the use of CSAV from small group to collectives. Future research will be devoted to explore the use of CSAV and other form of visual analytics to improve users' engagement and sensemaking of large-scale online deliberation processes.

Acknowledgements. The authors thank the support of Catalyst (FP7 program - grant agreement \#6111188) under which this work has been finalised. 


\section{Reference}

1. Shirky, C.: Here Comes Everybody: How change happens when people come together (2009)

2. Sunstein, C.R.: Infotopia: How Many Minds Produce Knowledge. Infotopia: How Many Minds Produce Knowledge (2006)

3. Klein, M., Iandoli, L.: Supporting Collaborative Deliberation Using a Large-Scale Argumentation System: The MIT Collaboratorium (2008)

4. Kunz, W., Rittel, H.W.J.: Issues as elements of information systems (1970)

5. OECD: Promise and Problems of E-Democracy: Challenges of Online Citizen Engagement. 1-162 (2003)

6. Benn, N., Macintosh, A.: Argument Visualization for eParticipation: Towards a Research Agenda and Prototype Tool. In: Tambouris, E., Macintosh, A., de Bruijn, H. (eds.) ePart 2011. LNCS, vol. 6847, pp. 60-73. Springer, Heidelberg (2011)

7. Gordon, T., Macintosh, A., Renton, A.: DEMO-net: D5. 2: Argumentation Support Systems, http://itc.napier.ac.uk

8. Renton, A., Macintosh, A.: Computer-Supported Argument Maps as a Policy Memory. The Information Society 23, 125-133 (2007)

9. Efthimios Tambouris, N.L.A.K.T.: A Framework for Assessing eParticipation Projects and Tools, pp. 1-10 (2006)

10. Buckingham Shum, S.B.: The roots of computer supported argument visualization. Visualizing argumentation (2003)

11. Engelbart, D.C.: Conceptual Framework for the Augmentation of Manl's Intellect (1963)

12. Novak, J.D.: Learning, creating, and using knowledge: Concept maps as facilitative tools in schools and corporations (2010)

13. Conklin, J., Begeman, M.L.: gIBIS: a hypertext tool for exploratory policy discussion. ACM Trans. Inf. Syst. 6, 303-331 (1988)

14. Horn, R.E.: Visual language and converging technologies in the next 10-15 years (and beyond). In: Proceedings of the 10th WSEAS International Conference on Applied Informatics and Communications (2002)

15. Halasz, F.G., Moran, T.P., Trigg, R.H.: Notecards in a nutshell. Presented at the CHI 1987: Proceedings of the SIGCHI/GI Conference on Human Factors in Computing Systems and Graphics Interface (April 1987)

16. Schuler, W., Smith, J.B.: Author's Argumentation Assistant (AAA): a hypertext-based authoring tool for argumentative texts. Hypertext: concepts, systems and applications (1992)

17. Shipman III, F.M., Marshall, C.C.: Formality Considered Harmful: Experiences, EmergingThemes, and Directions on the Use of Formal Representations inInteractive Systems. Computer Supported Cooperative Work 8 (1999)

18. Fischer, G., Lemke, A.C., McCall, R., Morch, A.I.: Making Argumentation Serve Design. Human-Computer Interaction 6, 393-419 (1991)

19. Buckingham Shum, S., Hammond, N.: Argumentation-based design rationale: what use at what cost? International Journal of Human-Computer Studies 40 (1994)

20. Simon, S., Erduran, S., Osborne, J.: Enhancing the quality of argumentation in school science. Journal of Research in Science Teaching 41(10), 994-1020 (2004)

21. Macintosh, A.: The emergence of digital governance. Significance (2008)

22. Macintosh, A.: E-democracy and e-participation research in Europe. Digital Government (2008)

23. Klein, M.: The MIT Collaboratorium: Enabling Effective Large-Scale Deliberation for Complex Problems (2007) 
24. Scheuer, O., Loll, F., Pinkwart, N., McLaren, B.M.: Computer-supported argumentation: A review of the state of the art. International Journal of Computer-Supported Collaborative Learning 5, 43-102 (2010)

25. Suthers, D.D.: Collaborative representations: Supporting face to face and online knowledge-building discourse. System Sciences (2001)

26. Suthers, D., Weiner, A., Connelly, J.: Belvedere: Engaging students in critical discussion of science and public policy issues. In: Proceedings of the 7th World Conference on Artificial Intelligence in Education (1995)

27. De Groot, R., Drachman, R., Hever, R.: CSCL 2007: Proceedings of the 8th Iternational Conference on Computer supported Collaborative Learning (2007)

28. Gordon, T.F., Prakken, H., Walton, D.: The Carneades model of argument and burden of proof. Artificial Intelligence 171 (2007)

29. Van Gelder, T.: The rationale for Rationale ${ }^{\mathrm{TM}}$. Law (2007)

30. Bell, P.: Using argument representations to make thinking visible for individuals and groups. Presented at the Proceedings of the 2nd International Conference on Computer Support for Collaborative Learning, Toronto, Ontario, Canada (1997)

31. Linn, M.C., Clark, D., Slotta, J.D.: WISE design for knowledge integration. Sci. Ed. 87, 517-538 (2003)

32. Conklin, J.E.: Conklin: "Designing organizational memory: preserving intellectual assets in a knowledge economy". Group Decision Support Systems 1, 362 (1996)

33. Buckingham Shum, S., Selvin, A.M., Sierhuis, M., Conklin, J., Haley, C.B., Nuseibeh, B.: Hypermedia Support for Argumentation-Based Rationale. Presented at the, Berlin, Heidelberg (2006)

34. De Liddo, A., Buckingham Shum, S., Convertino, G., Sándor, Á., Klein, M.: Collective intelligence as community discourse and action. Presented at the CSCW 2012: Proceedings of the ACM 2012 Conference on Computer Supported Cooperative Work Companion (February 2012)

35. Buckingham Shum, S.: Cohere: Towards Web 2.0 Argumentation. Presented at the Proceeding of the 2008 Conference on Computational Models of Argument: Proceedings of COMMA 2008 (June 2008)

36. Rahwan, I., Zablith, F., Reed, C.: Laying the foundations for a world wide argument web. Artificial Intelligence (2007)

37. MacLean, A., Young, R.M., Bellotti, V.M.E., Moran, T.P.: Questions, options, and criteria: elements of design space analysis. Human-Computer Interaction 6 (1991)

38. Karacapilidis, N., Tzagarakis, M., Karousos, N.: Tackling cognitively-complex collaboration with CoPe_it! International Journal of Web-Based Learning and Teaching Technologies (IJWLTT) 4(3), 22-38 (2009)

39. Hair, D.C.: LEGALESE: a legal argumentation tool. SIGCHI Bulletin 23 (1991)

40. Hill, W.C., Hollan, J.D., Wroblewski, D., McCandless, T.: Edit wear and read wear. Presented at the CHI 1992: Proceedings of the SIGCHI Conference on Human Factors in Computing Systems (June 1992)

41. Glaser, B.G., Strauss, A.L., Strutzel, E.: The discovery of grounded theory; strategies for qualitative research. Nursing Research (1968)

42. Brown, E., Cairns, P.: A grounded investigation of game immersion. In: CHI 2004 extended Abstracts on Human Factors in Computing Systems. ACM (2004)

43. De Liddo, A., Buckingham Shum, S.: The Evidence Hub: harnessing the collective intelligence of communities to build evidence-based knowledge (2013)

44. McCall, R.J.: PHI: A conceptual foundation for design hypermedia. Design Studies (1991) 